\title{
Impact of COVID-19 lockdown on the metabolic control parameters in patients with diabetes mellitus: a systematic review and meta-analysis
}

\author{
Ifan Ali $W^{1}$, Nando Reza $\mathrm{P}^{1}$, Nurizzah Farahiyah $\mathrm{S}^{1}$, Elsha Stephanie $\mathrm{A}^{1}$, Tiffany $\mathrm{K}^{1}$, \\ Maharani Ayuputeri $W^{1}$, M Rifqi $W^{1}$, Lilik Djuari ${ }^{2}$, Hermina Novida ${ }^{3}$
}

${ }^{1}$ Faculty of Medicine, Airlangga University, Indonesia

${ }^{2}$ Department of Public Health and Preventive Medicine, Airlangga University, Indonesia

${ }^{3}$ Department of Internal Medicine, Airlangga University, Indonesia

\section{Correspondence:}

Hermina Novida, MD, $\mathrm{PhD}$

Department of Internal Medicine, Airlangga University

Jalan Mayjen Prof. Dr. Moestopo No.47, Surabaya, Indonesia

E-mail address: hermina-n@fk.unair.ac.id

\begin{abstract}
Background: The abrupt implementation of COVID-19 lockdown had impacted the management of diabetes mellitus. Limited access to health facilities and alterations in daily lifestyle put metabolic control among patients at risk. Thus, we observed the differences in metabolic control parameters in diabetic patients before and during the lockdown.
\end{abstract}

Methods: We performed searches from five databases. Meta-analyses were done using random or fixed-effect approaches with glycemic control parameters (HbA1c, RBG, FBG, TIR, TAR, TBR) as primary outcomes. Mean difference (MD), Confidence Interval (CI), and $p$-value were calculated. Lipid profile presented as secondary outcome.

Results: 21 studies with 3992 diabetic patients were included in the study. Meta-analysis presented an increase on HbA1c of T1D and T2D patients $[\mathrm{MD}=+0.06 \%(95 \% \mathrm{CI}-0.10$ $\left.0.23), \mathrm{I}^{2}=77 \%, \mathrm{p}=0.45\right]$, TBR of T1D patients $\left[\mathrm{MD}=-0.05 \%(95 \% \mathrm{CI}-0.38-0.28), \mathrm{I}^{2}=0 \%\right.$, $\mathrm{p}=0.77]$, FBG of T2D patients $\left[\mathrm{MD}=+3,47 \mathrm{mg} / \mathrm{dL}(95 \% \mathrm{CI} 1.22-5.73), \mathrm{I}^{2}=0 \%, \mathrm{p}=0.002\right]$, and lipid profile on qualitative analysis. However, TIR, TAR, and RBG of T1D patients were improved $\left[\mathrm{MD}=+3.52 \%(95 \% \mathrm{CI} 0.29-6.74), \mathrm{I}^{2}=76 \%, \mathrm{p}=0.03 ; \mathrm{MD}=-3.36 \%(95 \% \mathrm{CI}\right.$ $-6.48-0.25), \mathrm{I}^{2}=75 \%, \mathrm{p}=0.03 ; \mathrm{MD}=-0.91 \mathrm{mg} / \mathrm{dL}$ (95\%CI $\left.\left.-4.52-6.34\right), \mathrm{I}^{2}=88 \%, \mathrm{p}=0.74\right]$.

Conclusion: Lockdown enforcement did not worsen the glycemic control parameter in diabetic patients. Particular parameters inclined to improve during the lockdown.

\section{Keywords}

COVID-19 lockdown, Diabetes mellitus, Meta-analysis, Metabolic control, Systematic review. 


\section{INTRODUCTION}

Since COVID-19 first emerged in China, the transmission of the virus has continued worldwide despite the intense preventive efforts as the lockdown that has been carried out by various countries worldwide [1]. Lockdown is defined as a restrictive policy to force people to stay at home or postpone unnecessary travel due to the spread of a dangerous disease [2]. Lockdown is considered the last in five steps of public health actions: prevention, protection, restriction, controlling, and lockdown. The policy applied as the transmission of COVID-19 went south with a rapid increase in morbidities and mortalities in several countries [3]. A study in India stated that lockdown is a practical decision to control COVID-19 transmission and provides time for the government and hospitals to develop proper management. Lockdown is vital in the track and trace strategy of this pandemic. In healthcare services, precautions include modification of medical appointments into virtual consultations. Thus, the rush of patients could be avoided and the exposures to COVID-19 could be minimized in non-emergency situations [4].

Lockdown had given rise to significant changes in daily life, including a decrease in physical activity and increases in sedentary lifestyle and dietary changes. Short supply and limited access to fresh and healthy food had led to increased preserved and fastfood consumption. Psychological and emotional states were also affected, triggering dysfunctional eating behavior and appetite dysregulation in particular individuals [5-8].

During the lockdown, vulnerable populations such as patients with chronic diseases tend to encounter restrictions in healthcare access, including routine medical engagements and procuring medications [9]. Indirect communications and virtual consultations tend to provide substandard care for the patients. As healthcare facilities had to deal with limited human and supply resources, reminders and daily routine assistance had also been diminished. Such difficulties would be troublesome in chronic disease management such as diabetes mellitus. Routine monitoring of metabolic control parameters, including glycemic and lipid control, was significantly affected $[10,11]$.

Poor clinical outcomes had been reported among COVID-19 patients with comorbid conditions. Prior studies stated that diabetes is one of the independent predictors of intensive care requirement, ventilation, or even death in COVID-19 patients. An uncontrolled lipid profile could also increase cardiovascular risks in diabetic patients $[12,13]$. Therefore, diabetes should be a concern amidst the pandemic, notably during the lockdown period, to prevent an increase in vulnerability to COVID-19 as well as the morbidity and mortality of the disease [14-16].

Through this systematic review and meta-analysis, we ought to determine the impact of COVID-19 lockdown on metabolic control, including glycemic control and lipid control among diabetic patients. Observation in metabolic parameter alterations before and during the lockdown could be a reference in advancing the care and management of diabetic patients during these situations.

\section{MATERIALS AND METHODS}

This study was performed in accordance with the Preferred Reporting Items for Systematic Reviews and Meta-Analyses (PRISMA) 2020 reporting guideline [17]. The study protocol has been registered in the PROSPERO database (CRD42021252321) [18].

\section{Search strategy}

A comprehensive electronic-based literature search was conducted in PubMed, ScienceDirect, Cochrane Library, JSTOR, and DOAJ databases from April 19-26, 2021, for studies published in English. Medical Subject Heading (MeSH) terms was used combined with the Boolean operator (AND/OR) with the following keywords: (COVID) AND ((pandemic) OR 
(lockdown) OR (quarantine)) AND (diabetes mellitus [MeSH Terms]) AND ((glycemic control) OR (glucose level) OR (lipid profile)). The authors also performed manual searches to retrieve potentially relevant articles from the reference list of retrieved articles.

\section{Study selection}

Title and abstract screening were done on each article, following duplication removal using the Mendeley reference manager. Two authors (IAW and NRP) independently conducted the screening based on the following eligibility criteria: observational studies (cross-sectional, case-control, or cohort), published between 2020 and 2021, including patients of any age with type 1 (T1D) or type 2 diabetes (T2D), reported the glycemic control parameters and/or lipid profile as primary or secondary outcomes, and assessed comparison of metabolic control among diabetic patients during and before lockdown. We excluded studies with the following criteria: experimental studies, non-English articles, included other types of diabetes, involved patients with SARS CoV-2 comorbid infection, and not reported the glycemic control and/or lipid profile during the lockdown. The searching, selecting, and screening process was recorded and documented in the PRISMA flow diagram (Figure 1).

\section{Data extraction}

Three independent authors (IAW, NRP, and NFS) used a standardized form to extract the data. Any discrepancies were resolved by discussion among authors until reaching a consensus. The following data were extracted: first author name, year of publication, the country setting, study design, sample size, study period, age, and types of diabetes. Glycemic control parameters (HbA1c or estimated A1c (eA1c), random blood glucose (RBG), fasting blood glucose (FBG), time-in-range (TIR), time-above-range (TAR), and time-below-range (TBR), lipid control parameters (total cholesterol (TC), low-density lipoprotein (LDL), high-density lipopreotein (HDL), and triglyceride (TG), measurement methods, and comparison of metabolic control before and during COVID-19 lockdown were also extracted.

\section{Quality assessment}

Quality assessment was performed independently by two authors (IAW and NFS). Any discrepancies were resolved by discussion among authors until reaching a consensus. The quality assessment of included studies was performed with the Effective Public Health Practice Project (EPHPP) tool, consisting of six components: (i) selection bias, (ii) study design, (iii) confounders, (iv) blinding, (v) data collection method, and (vi) withdrawals and dropouts. Each component rated as 1 (strong), 2 (moderate), or 3 (weak). Based on the weak rating counts, the global rating of each study was assigned as strong, moderate, or weak quality articles [19].

\section{Operational definitions}

TIR, TAR, and TBR defined as the percentage of time in a day a person spends the blood glucose levels respectively in a target range $(70-180 \mathrm{mg} / \mathrm{dL}$ [3.9-10.0 $\mathrm{mmol} / \mathrm{L}])$, above range $(>180 \mathrm{mg} / \mathrm{dL})$, and below range $(<70 \mathrm{mg} / \mathrm{dL})[20]$.

\section{Data synthesis and analysis}

The primary outcomes of this study were the difference in glycemic control parameters before and during the lockdown: HbA1c, RBG, and FBG; along with other glycemic control parameters obtained from the continuous glucose monitoring (CGM) or flash glucose monitoring (FGM) device: TIR, TAR, and TBR. Secondary outcomes were the difference in lipid control parameters during and before the lockdown: TC, LDL, HDL, and TG. The mean and standard deviation of the glycemic control parameters and lipid profile data from each study were extracted. Any outcomes presented as a median and interquartile range (IQR) were not included in the meta-analysis. The comparison of glycemic control parameters and lipid 
profile during and before the lockdown were analyzed as continuous variables using the mean difference (MD) and summarized with their corresponding 95\%CI. The overall effect (Z test) was considered significant if the $p$-value was $<0.05$. Heterogeneity was assessed using the Q statistic and $\mathrm{I}^{2}$ test. The $\mathrm{I}^{2}$ test presents the variability percentage of the analysis due to clinical or methodological heterogeneity rather than sampling error. The random-effects model was used when the $\mathrm{I}^{2}$ was $>50 \%$ or the $P$-value was $<0.1$; otherwise, the fixed-effects model was preferred. Potential publication bias was observed visually using Begg's funnel plots. If such bias was evident, the trim-and-fill method was performed to generate a pooled estimate that accounted for the unpublished findings. We preplanned to perform subgroup analyses for all outcomes based on the study design. Sensitivity analyses were also performed based on the quality of the studies. All statistical analyses were performed using Review Manager version 5.4 (The Cochrane Collaboration).

\section{RESULTS}

The search strategy yielded 166 articles from primary databases and manual searching after removing four duplications using Mendeley reference manager. Once titles and abstracts were screened, 45 articles were selected for full-text evaluation. Of those, 21 studies with 3992 participants met the eligibility criteria and were included in this study (Figure 1). Quality assessment resulted in twelve "strong" quality articles, eight "moderate" quality articles, and one "weak" quality article based on EPHPP tool (see Supplementary Material). Funnel plot asymmetry was obtained in HbA1c, RBG, TIR, and TAR outcomes (Figure 3).

All studies included were observational studies, consisting of thirteen cohort studies, five case-control studies, and three cross-sectional studies. Most studies were conducted in Italy, with others being conducted in Spain, France, Greece, Netherlands, Israel, Saudi Arabia, Turkey, and India. The characteristics of the included studies are presented in Table 1.

According to the 21 studies' findings, seven studies reported negative impacts and deterioration in glycemic control during the lockdown, while others reported no significant differences. However, some studies showed significant improvements in glycemic control among diabetic patients during the lockdown. Due to insufficient outcome data, only seventeen studies were included in the meta-analysis. The lipid control parameters were analyzed as secondary outcomes and presented as descriptive analysis since only two available studies reported the outcome. 


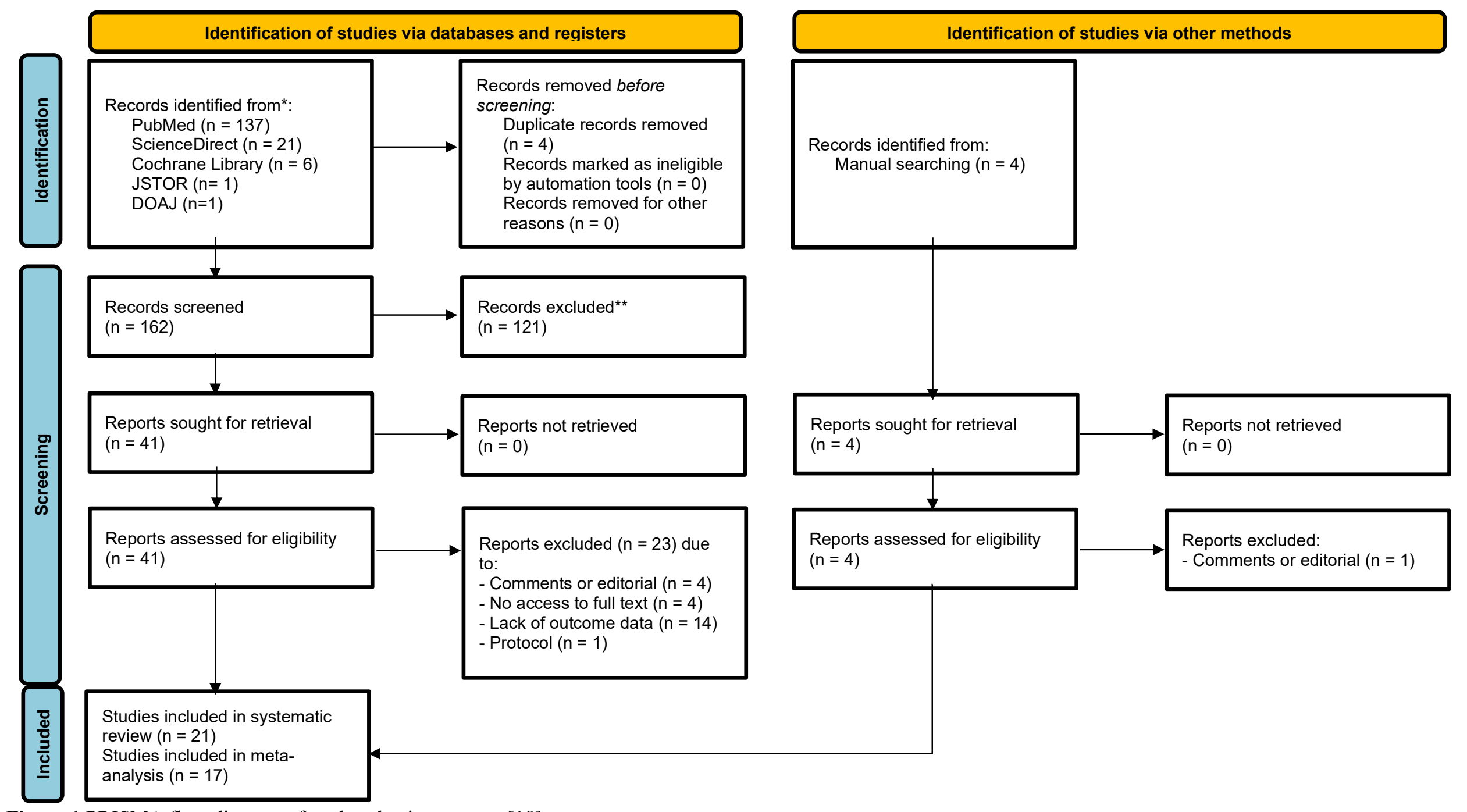

Figure 1 PRISMA flow diagram of study selection process [18] 
Table 1. Characteristics of the included study

\begin{tabular}{|c|c|c|c|c|c|c|c|c|c|}
\hline $\begin{array}{l}\text { Author (Year) } \\
\text { Country }\end{array}$ & $\begin{array}{l}\text { Study } \\
\text { design }\end{array}$ & $\begin{array}{l}\text { Sample } \\
\text { size }\end{array}$ & Study Period & $\begin{array}{c}\text { Diabetes } \\
\text { type }\end{array}$ & $\begin{array}{c}\text { Ages } \\
\text { Mean } \pm \text { SD } / \\
\text { Median (IQR) } \\
\end{array}$ & Outcomes & $\begin{array}{l}\text { Measurement } \\
\text { method }\end{array}$ & $\begin{array}{c}\text { Quality } \\
\text { assessment }\end{array}$ & Findings \\
\hline $\begin{array}{l}\text { Al-agha et al. (2021) } \\
\text { Saudi Arabia [30] }\end{array}$ & Cross-sectional & 150 & Apr - Jun 2020 & T1D & $12.45 \pm 4.62$ & RBG, $\mathrm{HbAlc}$ & SMBG & Strong & $\begin{array}{l}\text { Lockdown affected RBG and BMI } \\
\text { due to lack of physical activity, } \\
\text { increased consumption of } \\
\text { carbohydrates and fast food, and } \\
\text { worsening mood. }\end{array}$ \\
\hline $\begin{array}{l}\text { Alaqeel et al. (2021) } \\
\text { Saudi Arabia [21] }\end{array}$ & $\begin{array}{l}\text { Retrospective } \\
\text { cohort }\end{array}$ & $\begin{array}{c}154 \text { pre- } \\
\text { lockdown } \\
106 \\
\text { lockdown }\end{array}$ & $1 \mathrm{Mar}-30$ Jun 2020 & T1D & $9.8 \pm 0.2$ & $\mathrm{HbA1c}$ & SMBG & Strong & $\begin{array}{l}\text { Lockdown increased the frequency } \\
\text { of DKA in pediatric T1D patients. }\end{array}$ \\
\hline $\begin{array}{l}\text { Aragona et al. (2020) } \\
\text { Italy [22] }\end{array}$ & $\begin{array}{l}\text { Retrospective } \\
\text { cohort }\end{array}$ & 63 & $\begin{array}{c}\text { Pre-lockdown: } \\
21 \text { Feb - 6 Mar } 2020 \\
\text { Lockdown: } \\
\text { Early } 11-25 \text { Mar; } \\
\text { Mid } 11-25 \text { Apr } 2020 \\
\end{array}$ & T1D & $44.0 \pm 12.0$ & $\begin{array}{c}\text { RBG, eAlc, } \\
\text { TIR, TAR, } \\
\text { TBR }\end{array}$ & FGM, CGM & Strong & $\begin{array}{c}\text { Glycemic control was significantly } \\
\text { improved due to an increase in daily } \\
\text { routine activities and a decrease in } \\
\text { work-related stress. }\end{array}$ \\
\hline $\begin{array}{l}\text { Barchetta et al. (2020) } \\
\text { Italy [32] }\end{array}$ & $\begin{array}{l}\text { Retrospective } \\
\text { cohort }\end{array}$ & 50 & $\begin{array}{c}\text { Pre-lockdown: } \\
\text { 20 Jan - 3 Feb 2020 } \\
\text { Lockdown: } \\
28 \text { Mar-11 Apr 2020 }\end{array}$ & T1D & $40.7 \pm 13.5$ & $\begin{array}{c}\text { RBG, eAlc, } \\
\text { TIR, TAR, } \\
\text { TBR }\end{array}$ & FGM, CGM & Moderate & $\begin{array}{l}\text { Lockdown significantly deteriorated } \\
\text { glycemic control of T1D patients. } \\
\text { Work instability impaired glycemic } \\
\text { variability. }\end{array}$ \\
\hline $\begin{array}{l}\text { Biancalana et al. (2020) } \\
\text { Italy [23] }\end{array}$ & $\begin{array}{l}\text { Prospective } \\
\text { cohort }\end{array}$ & 114 & 9 Mar - 4 May 2020 & $\mathrm{~T} 2 \mathrm{D}$ & $69.4 \pm 10.3$ & $\begin{array}{l}\text { HbA1c, FBG, } \\
\text { TC, LDL, TG }\end{array}$ & SMBG & Strong & $\begin{array}{l}\text { Lockdown caused short-term } \\
\text { worsening in glycemic control and } \\
\text { lipid profile in } 1 / 4 \text { of well-controlled } \\
\text { T2D patients. Pre-lockdown }\end{array}$ \\
\hline
\end{tabular}


triglyceride level is the sole predictor of lockdown glycemic control.

\begin{tabular}{|c|c|c|c|c|c|c|c|c|c|}
\hline $\begin{array}{l}\text { Bonora et al. (2020) } \\
\text { Italy [33] }\end{array}$ & $\begin{array}{l}\text { Retrospective } \\
\text { cohort }\end{array}$ & 20 & $\begin{array}{c}\text { Pre-lockdown: } \\
1 \text { week before } \\
\text { outbreak SARS-CoV- } \\
2 \text { in Italy } \\
\\
\text { Lockdown: } \\
1^{\text {st }} \text { week of lockdown }\end{array}$ & $\mathrm{T} 1 \mathrm{D}$ & $36.9 \pm 13.4$ & $\begin{array}{l}\text { RBG, TIR, } \\
\text { TAR, TBR }\end{array}$ & FGM & Moderate & $\begin{array}{l}\text { Short-term glycemic control was } \\
\text { improved in T1D patients with good } \\
\text { control who remained at home } \\
\text { during lockdown due to a slowdown } \\
\text { or decrease in daily routine activities } \\
\text { despite limited activities and } \\
\text { psychological stress. Long term } \\
\text { effects require further investigation. }\end{array}$ \\
\hline $\begin{array}{l}\text { Brener et al. (2020) } \\
\text { Israel [34] }\end{array}$ & $\begin{array}{l}\text { Retrospective } \\
\text { cohort }\end{array}$ & 102 & $\begin{array}{c}\text { Pre-lockdown: } \\
23 \text { Feb - 7 Mar } 2020 \\
\text { Lockdown: } \\
25 \text { Mar - } 7 \text { Apr } 2020\end{array}$ & $\mathrm{~T} 1 \mathrm{D}$ & $10.9 \pm 3.9$ & $\begin{array}{l}\text { RBG, TIR, } \\
\text { TAR, TBR }\end{array}$ & CGM & Strong & $\begin{array}{l}\text { Glycemic control during lockdown } \\
\text { is relatively stable in pediatric T1D } \\
\text { patients. Interventions should be } \\
\text { focused on young patients in low } \\
\text { socioeconomic populations. }\end{array}$ \\
\hline $\begin{array}{l}\text { Christoforidis et al. (2020) } \\
\text { Greece [35] }\end{array}$ & Case-control & 34 & $\begin{array}{c}\text { Pre-lockdown: } \\
3 \text { weeks to } 10 \text { Mar } \\
2020 \\
\text { Lockdown: } \\
3 \text { weeks after } 11 \text { Mar } \\
2020 \\
\end{array}$ & $\mathrm{~T} 1 \mathrm{D}$ & $11.37 \pm 4.45$ & $\begin{array}{l}\text { RBG, TIR, } \\
\text { TAR, TBR }\end{array}$ & HCL system & Moderate & $\begin{array}{l}\text { Glycemic control during lockdown } \\
\text { in pediatric T1D patients could be } \\
\text { stabilized using insulin pumps with } \\
\text { blood glucose monitoring sensors. }\end{array}$ \\
\hline $\begin{array}{l}\text { Cotovad-Bellas et al. (2020) } \\
\text { Spain [26] }\end{array}$ & Case-control & 44 & $\begin{array}{c}\text { Pre-lockdown: } \\
1-14 \text { Mar } 2020 \\
\text { Lockdown: } \\
6-19 \text { Apr } 2020\end{array}$ & $\mathrm{~T} 1 \mathrm{D}$ & $37.0 \pm 18.0$ & $\begin{array}{l}\text { RBG, eA1c, } \\
\text { TIR, TAR, } \\
\text { TBR }\end{array}$ & FGM, CGM & Moderate & $\begin{array}{l}\text { No disruptive effect on glycemic } \\
\text { control during lockdown due to } \\
\text { increased time for exercise and } \\
\text { home-made cooking. }\end{array}$ \\
\hline $\begin{array}{l}\text { Di Dalmazi et al. (2020) } \\
\text { Italy [40] }\end{array}$ & $\begin{array}{l}\text { Prospective } \\
\text { cohort }\end{array}$ & 130 & $\begin{array}{c}\text { Pre-lockdown: } \\
20 \text { Feb - 10 Mar } 2020\end{array}$ & $\mathrm{~T} 1 \mathrm{D}$ & $\begin{array}{c}\text { Children: } \\
8.8(7.7-10.6) \\
\text { Adolescent: } \\
15.6(14.2- \\
16.8) \\
\text { Adult: } \\
45(29-58.1)\end{array}$ & $\begin{array}{l}\text { RBG, eAlc, } \\
\text { TIR, TAR, } \\
\text { TBR }\end{array}$ & CGM & Moderate & $\begin{array}{l}\text { Glycemic control in T1D patients } \\
\text { did not worsen in adults during } \\
\text { lockdown period and both children } \\
\text { and adults during post-lockdown } \\
\text { period due to the use of CGM, } \\
\text { physical activity, and stress } \\
\text { reduction. No changes observed in } \\
\text { the adolescents. }\end{array}$ \\
\hline
\end{tabular}




\begin{tabular}{|c|c|c|c|c|c|c|c|c|c|}
\hline $\begin{array}{l}\text { Dover et al. (2020) } \\
\text { United Kingdom [44] }\end{array}$ & $\begin{array}{l}\text { Prospective } \\
\text { cohort }\end{array}$ & 572 & $\begin{array}{c}\text { Pre-lockdown: } \\
11-18 \text { Mar } 2020 \\
\text { Lockdown: } \\
14-21 \text { May } 2020\end{array}$ & T1D & $39(31-50)$ & $\begin{array}{l}\text { RBG, eA1c, } \\
\text { TIR, TAR, } \\
\text { TBR }\end{array}$ & FGM & Weak & $\begin{array}{l}\text { Glycemic control in T1D patients } \\
\text { did not worsen during lockdown. } \\
\text { Socioeconomic declines could } \\
\text { increase the risk of poor glycemic } \\
\text { control. }\end{array}$ \\
\hline $\begin{array}{l}\text { Karatas et al. (2021) } \\
\text { Turkey [27] }\end{array}$ & Case-control & 85 & Mar 2019 - Oct 2020 & $\mathrm{~T} 2 \mathrm{D}$ & $54.81 \pm 10.53$ & $\begin{array}{l}\text { HbAlc, FBG, } \\
\text { LDL, HDL, } \\
\text { TG }\end{array}$ & SMBG & Strong & $\begin{array}{l}\text { Prolongation of lockdown worsened } \\
\text { glycemic control and increased } \\
\text { triglyceride levels in T2D patients, } \\
\text { which was not associated with } \\
\text { weight gain. }\end{array}$ \\
\hline $\begin{array}{l}\text { Khare and Jindal (2020) } \\
\text { India [37] }\end{array}$ & $\begin{array}{l}\text { Prospective } \\
\text { cohort }\end{array}$ & 143 & $\begin{array}{l}\text { The first } 3 \text { weeks of } \\
\text { lockdown }\end{array}$ & $\mathrm{T} 2 \mathrm{D}$ & $54.68 \pm 9.22$ & FBG, PPBG & SMBG & Strong & $\begin{array}{l}\text { Glycemic control was impaired } \\
\text { during the 3-week lockdown period } \\
\text { due to lifestyle changes, } \\
\text { psychological stress, and difficulties } \\
\text { in access to treatments and health } \\
\text { care facilities. }\end{array}$ \\
\hline & & & $\begin{array}{c}\text { Pre-lockdown: } \\
23 \text { Feb-8 Mar } 2020\end{array}$ & & & & & & \\
\hline $\begin{array}{l}\text { Longo et al. (2020) } \\
\text { Italy [39] }\end{array}$ & $\begin{array}{l}\text { Retrospective } \\
\text { cohort }\end{array}$ & 30 & $\begin{array}{c}\text { Lockdown: } \\
\text { Early } 9-23 \text { Mar } \\
2020 \\
\text { End } 20 \mathrm{Apr}-3 \text { May } \\
2020 \\
\end{array}$ & T1D & $31.5(25-42)$ & $\begin{array}{l}\text { RBG, eA1c, } \\
\text { TIR, TAR, } \\
\text { TBR }\end{array}$ & HCL system & Moderate & $\begin{array}{l}\text { Glycemic control during lockdown } \\
\text { significantly improved in adult T1D } \\
\text { patients using HCL. }\end{array}$ \\
\hline $\begin{array}{l}\text { Mesa et al. (2020) } \\
\text { Spain [28] }\end{array}$ & Case-control & 92 & $\begin{array}{c}\text { Pre-lockdown: } \\
\text { 23 Feb-7 Mar } 2020 \\
\text { Lockdown: } \\
1-14 \text { Apr } 2020 \\
\end{array}$ & T1D & $42.8 \pm 13.9$ & $\begin{array}{l}\text { RBG, eAlc, } \\
\text { TIR, TAR, } \\
\text { TBR }\end{array}$ & CGM & Strong & $\begin{array}{l}\text { Glycemic control was improved } \\
\text { without hypoglycemic tendencies in } \\
\text { T1D patients during lockdown due } \\
\text { to strict daily routines at home. }\end{array}$ \\
\hline $\begin{array}{l}\text { Parise et al. (2021) } \\
\text { Italy [24] }\end{array}$ & $\begin{array}{l}\text { Prospective } \\
\text { cohort }\end{array}$ & 166 & $10 \mathrm{Mar}-3$ June 2020 & T1D & $40.0 \pm 14.0$ & $\begin{array}{l}\text { RBG, eAlc, } \\
\text { TIR, TAR, } \\
\text { TBR }\end{array}$ & CGM & Strong & $\begin{array}{l}\text { Structured virtual visits } \\
\text { (teleassistance) could help maintain } \\
\text { and improve glycemic control in } \\
\text { T1D patients during lockdown. }\end{array}$ \\
\hline $\begin{array}{l}\text { Potier et al. (2021) } \\
\text { France [36] }\end{array}$ & Cross-sectional & 1378 & $23-28$ Apr 2020 & T1D & $45.6 \pm 13.6$ & RBG & FGM & Strong & $\begin{array}{c}\text { Glycemic control was improved } \\
\text { during lockdown due to improved } \\
\text { diet, increased physical activity, and } \\
\text { increased FGM scans. }\end{array}$ \\
\hline
\end{tabular}




\begin{tabular}{|c|c|c|c|c|c|c|c|c|c|}
\hline \multirow{2}{*}{$\begin{array}{l}\text { Ruissen et al. (2021) } \\
\text { Netherlands [25] }\end{array}$} & \multirow{2}{*}{$\begin{array}{l}\text { Prospective } \\
\text { cohort }\end{array}$} & \multirow{2}{*}{435} & $\begin{array}{c}\text { Pre-lockdown: } \\
\text { 24 Feb - } 8 \text { Mar } 2020 \\
\end{array}$ & T1D & $50.1 \pm 14.9$ & \multirow{2}{*}{$\begin{array}{c}\text { HbAlc, TIR, } \\
\text { TAR, TBR }\end{array}$} & \multirow{2}{*}{ FGM, CGM } & \multirow{2}{*}{ Strong } & \multirow{2}{*}{$\begin{array}{l}\text { Increased stress and anxiety, weight } \\
\text { gain, and reduced physical activity, } \\
\text { did not worsen short-term glycemic } \\
\text { control in T1D and T2D patients } \\
\text { with good control. }\end{array}$} \\
\hline & & & $\begin{array}{c}\text { Lockdown: } \\
24 \text { Apr - } 7 \text { May } 2020\end{array}$ & $\mathrm{~T} 2 \mathrm{D}$ & $62.5 \pm 11.6$ & & & & \\
\hline \multirow{2}{*}{$\begin{array}{l}\text { Tornese et al. (2020) } \\
\text { France [41] }\end{array}$} & \multirow{2}{*}{$\begin{array}{l}\text { Retrospective } \\
\text { cohort }\end{array}$} & \multirow[b]{2}{*}{13} & $\begin{array}{c}\text { Pre-lockdown: } \\
10-23 \text { Feb } 2020\end{array}$ & \multirow[b]{2}{*}{ T1D } & \multirow{2}{*}{$\begin{array}{c}14.2(11.4- \\
15.5)\end{array}$} & \multirow{2}{*}{$\begin{array}{l}\text { RBG, eA1c, } \\
\text { TIR, TAR, } \\
\text { TBR }\end{array}$} & \multirow[b]{2}{*}{ HCL system } & \multirow[b]{2}{*}{ Moderate } & \multirow{2}{*}{$\begin{array}{l}\text { Glycemic control in adolescent T1D } \\
\text { patients during lockdown did not } \\
\text { worsen and tend to improve in } \\
\text { patients who maintained physical } \\
\text { activity. }\end{array}$} \\
\hline & & & $\begin{array}{c}\text { Lockdown: } \\
9-22 \text { Mar } 2020 \\
\end{array}$ & & & & & & \\
\hline $\begin{array}{l}\text { Verma et al. (2020) } \\
\text { India [31] }\end{array}$ & Cross-sectional & 52 & 25 Mar - 31 May 2020 & T1D & 11.9 & RBG, $\mathrm{HbAlc}$ & SMBG & Moderate & $\begin{array}{c}\text { Glycemic control of T1D patients } \\
\text { worsened during lockdown, mainly } \\
\text { due to the unavailability of } \\
\text { insulin/glucostrips. }\end{array}$ \\
\hline \multirow{2}{*}{$\begin{array}{l}\text { Viñals et al. (2020) } \\
\text { Spain [29] }\end{array}$} & \multirow{2}{*}{ Case-control } & \multirow{2}{*}{59} & $\begin{array}{c}\text { Pre-lockdown: } \\
\text { 23 Feb - } 7 \text { Mar } 2020\end{array}$ & \multirow{2}{*}{ T1D } & \multirow{2}{*}{$46.18 \pm 13.02$} & \multirow{2}{*}{$\begin{array}{l}\text { RBG, eAlc, } \\
\text { TIR, TAR, } \\
\text { TBR }\end{array}$} & \multirow{2}{*}{ HCL system } & \multirow{2}{*}{ Strong } & $\begin{array}{l}\text { Glycemic control in T1D patients } \\
\text { was maintained during lockdown }\end{array}$ \\
\hline & & & $\begin{array}{c}\text { Lockdown: } \\
1-14 \text { Apr } 2021\end{array}$ & & & & & & $\begin{array}{l}\text { without hypoglycemic tendencies } \\
\text { due to strict daily routine at home. }\end{array}$ \\
\hline
\end{tabular}

Abbreviations: T1D (type 1 diabetes); T2D (type 2 diabetes); CGM (continuous glucose monitoring); FGM (flash glucose monitoring); HCL (hybrid closed loop); SMBG (self-monitoring blood glucose); RBG (random blood glucose); PPBG (postprandial blood glucose); FBG (fasting blood glucose); eA1c (estimated A1c); TIR (time in range); TAR (time above range); TBR (time below range); TC (total cholesterol); LDL (low-density lipoprotein); HDL (high-density lipoprotein); TG (triglyceride) 


\section{Primary outcomes}

\section{Glycated Hemoglobin A1c (HbA1c)}

A total of eleven studies [21-31] with 1357 pre-lockdown and 1325 lockdown total participants, consisting of T1D and T2D patients, reported the mean level of HbA1c and estimated A1c which obtained either from CGM or FGM (Figure 2). A pooled estimate showed that lockdown did not significantly give rise to changes in HbAlc parameter $(\mathrm{MD}=0.06 \%$ [ $95 \% \mathrm{CI}-0.10$ to 0.23 ], $\mathrm{I}^{2}=77 \%$, random-effects). All HbA1c sub-group analysis presented no significant differences on $\mathrm{HbA} 1 \mathrm{c}$ between pre- and during lockdown on cohort studies $(\mathrm{MD}=-$ $0.01 \%$ [ $95 \% \mathrm{CI}-0.17$ to 0.14$], \mathrm{I}^{2}=65 \%$, random-effects), case-control ( $\mathrm{MD}=0.00 \%$ [ $95 \% \mathrm{CI}$ 0.32 to 0.32$], \mathrm{I}^{2}=75 \%$, random-effects), and cross-sectional study ( $\mathrm{MD}=0.56 \%$ [ $95 \% \mathrm{CI}-0.67$ to 1.78 ], $\mathrm{I}^{2}=95 \%$, random-effects).

\section{Random Blood Glucose (RBG)}

Twelve studies [22,24,26,28-36] on T1D patients reported mean difference of RBG with a total of 2162 pre-lockdown and 2210 lockdown participants (Figure 2). Pooled estimate showed no significant differences in RBG level between pre- and during lockdown period (MD=0.91 $\mathrm{mg} / \mathrm{dL}$ [ $95 \% \mathrm{CI}-4.52$ to 6.34$], \mathrm{I}^{2}=88 \%$, random-effects). RBG sub-group analysis presented no significant differences on cohort studies $(\mathrm{MD}=-0.71 \mathrm{mg} / \mathrm{dL}$ [95\%CI -7.64 to 6.22$], \mathrm{I}^{2}=0.64 \%$, random-effects) $[9,22,30-32]$, case-control studies ( $\mathrm{MD}=-2.47 \mathrm{mg} / \mathrm{dL}$ [95\%CI -8.91 to 3.98], $\mathrm{I}^{2}=75 \%$, random-effects) $[24,26,27,33]$, and cross-sectional studies ( $\mathrm{MD}=23.61 \mathrm{mg} / \mathrm{dL}(95 \% \mathrm{CI}$ -15.32 to $62.54, I^{2}=93 \%$, random-effects) $[28,29,34]$.

\section{Fasting Blood Glucose (FBG)}

Mean FBG level was reported in 2 cohort studies $[23,37]$ on T2D patients with a total of 257 participants on each arm (Figure 2). Meta-analysis showed a significant deterioration in FBG control from pre- to lockdown period ( $\mathrm{MD}=3.47 \mathrm{mg} / \mathrm{dL}[95 \% \mathrm{CI} 1.22$ to 5.73$], \mathrm{I}^{2}=0 \%$, fixedeffect).

\section{Time In Range (TIR)}

Data of mean level of TIR was reported in 8 studies [22,24,26,28,29,33-35] among T1D patients with a total of 580 participants on each arm (Figure 2). A significant improvement of TIR from pre- to during lockdown period was indicated (MD $=3.52 \%(95 \% \mathrm{CI} 0.29$ to 6.74 , $\mathrm{I}^{2}=76 \%$, random-effects). However, pooled estimates of subgroup analysis did not show significant improvement in TIR during lockdown on both cohort studies ( $\mathrm{MD}=4.61 \%[95 \% \mathrm{CI}$ -0.55 to 9.78$], \mathrm{I}^{2}=87 \%$, random-effects) and case-control studies ( $\mathrm{MD}=2.24 \%$ [95\%CI -0.43 to 4.90], $\mathrm{I}^{2}=0 \%$, fixed-effect).

\section{Time Above Range (TAR)}

Data of mean level of TAR was reported in 8 studies [22,24,26,28,29,33-35] among T1D patients with a total of 580 participants on each arm (Figure 2). The pooled effect showed that TAR was significantly improved from pre- to lockdown period (MD=-3.36\% [95\%CI -6.48 to $-0.25], I^{2}=75 \%$, random-effects). The result remained similar when this study was omitted. However, when subgroup analysis was performed, the pooled effects were failed to reach statistical significant on both cohort ( $\mathrm{MD}=-4.44 \%$ [95\%CI -9.83 to 0.94$], \mathrm{I}^{2}=86 \%$, randomeffects) and case-control studies ( $\mathrm{MD}=-2.20 \%$ [95\%CI -4.66 to 0.27$], \mathrm{I}^{2}=0 \%$, fixed-effect). 
a)

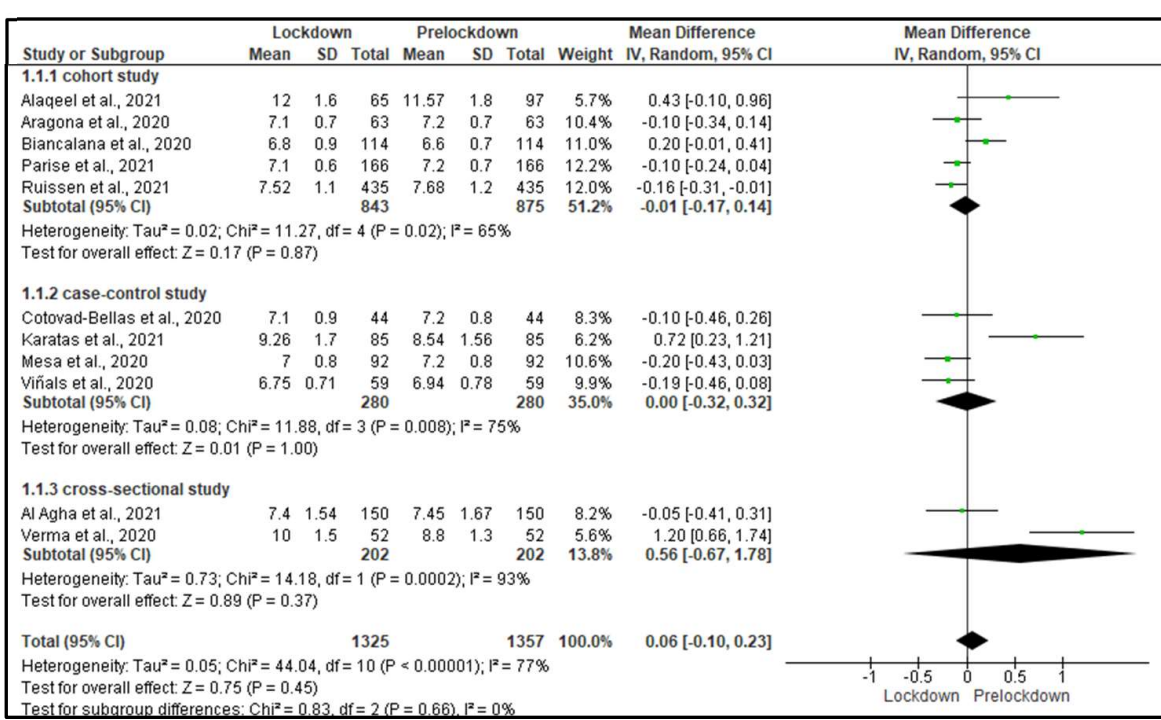

b)

\begin{tabular}{|c|c|c|c|c|c|c|c|c|c|}
\hline \multirow{3}{*}{$\begin{array}{l}\text { Study or Subgroup } \\
2.1 .1 \text { cohort study }\end{array}$} & \multicolumn{3}{|c|}{ Lockdown } & \multicolumn{3}{|c|}{$\begin{array}{l}\text { Prelockdown } \\
\end{array}$} & & \multirow{3}{*}{$\begin{array}{l}\text { Mean Difference } \\
\text { VV, Random, } 95 \% \mathrm{Cl}\end{array}$} & \multirow{2}{*}{$\begin{array}{l}\text { Mean Difference } \\
\mathrm{IV} \text {, Random, } 95 \% \mathrm{Cl}\end{array}$} \\
\hline & Mean & & & & & & Weight & & \\
\hline & & & & & & & & & \\
\hline $\begin{array}{l}\text { Aragona et at., } 202020 \\
\text { Barchetta et al., } 2020\end{array}$ & 165 & 25 & 50 & 154 & 15 & $\begin{array}{l}63 \\
50\end{array}$ & $\begin{array}{l}8.6 \% \\
9.6 \% \\
\end{array}$ & 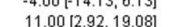 & \\
\hline 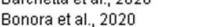 & 161 & 40.3 & 20 & 177.7 & 45.6 & 20 & & $-16.70-43.37,9.977$ & \\
\hline Brener et al., 2020 & 161.5 & 25.02 & & 163.4 & 24.04 & & $9.6 \%$ & $-1.90-1.94 .6 .614$ & \\
\hline Parise et al., 2021 & 159 & & 166 & 163 & & 166 & $10.7 \%$ & $-4.00[-9.82,1.82]$ & \\
\hline \multicolumn{10}{|c|}{$\begin{array}{l}\text { Heterogeneity: Tau }=36.36 ; \mathrm{Chi}^{2}=11.16 \text {, df }=4(P=0.02) ; \mathrm{P}^{2}=64 \% \\
\text { Test for overall effect: } Z=0.20(P=0.84)\end{array}$} \\
\hline \multicolumn{10}{|l|}{ 2.1.2 case-control study } \\
\hline $\begin{array}{l}\text { 2.1.2. case-contro Itudy } \\
\text { Christofididis et al., } 2020\end{array}$ & 184.47 & & 34 & & & & & $3.07[0.65,5.49]$ & \\
\hline Cotovad-Bellas et al., 2020 & 161 & 40 & 44 & 165 & 33 & 44 & $6.2 \%$ & $-4.00[-19.32,11.32]$ & \\
\hline Mesa et al., 2020 & 153.5 & 27 & 92 & 160.8 & & 92 & $9.5 \%$ & $-7.30[-15.65,1.05]$ & $\Rightarrow$ \\
\hline $\begin{array}{l}\text { Yinials etala.,2020 } \\
\text { Subtotal (95\% Cl) }\end{array}$ & & & $\begin{array}{r}59 \\
229\end{array}$ & & & $\begin{array}{r}59 \\
229\end{array}$ & $\begin{array}{l}9.9 .4 \% \\
37.4 \%\end{array}$ & $\begin{array}{l}-5.53[-12.9 .97,2.19] \\
-2.47[-8.91,3.98]\end{array}$ & ta \\
\hline \multicolumn{10}{|c|}{ 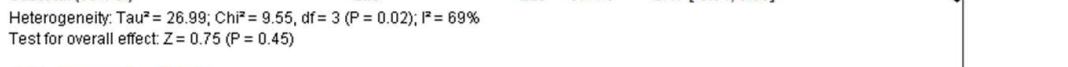 } \\
\hline \multirow{2}{*}{\multicolumn{10}{|c|}{ 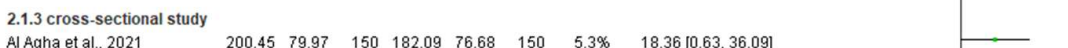 }} \\
\hline Al Agha et al., 2021 & 200.45 & & & & & & & & \\
\hline Potier et al., 2021 & 155.7 & 30.3 & 1378 & 163.5 & & 1378 & $11.9 \%$ & $-7.80[-10.10,-5.50]$ & - \\
\hline $\begin{array}{l}\text { Verma et ala., } 2020 \\
\text { Subtotola (1.5\% CII) }\end{array}$ & 276.9 & 64.7 & $\begin{array}{r}52 \\
1580\end{array}$ & 212.3 & 57.9 & $\begin{array}{r}52 \\
1580\end{array}$ & $\begin{array}{l}2 \\
0 \\
0\end{array} 2.0 .9 \%$ & $\begin{array}{r}64.60(411.00,88.20] \\
23.61[-1532,62.54]\end{array}$ & \\
\hline \multicolumn{10}{|c|}{$\begin{array}{l}\text { Heterogeneity : Tauz }=1110.42 ; C h \mathrm{P}^{2}=43.62 \text {, df }=2(P<0.00001) ; \quad F=95 \% \\
\text { Test for overall effect: } Z=1.19(P=0.23)\end{array}$} \\
\hline \multirow{2}{*}{\multicolumn{2}{|c|}{$\begin{array}{l}\text { Total }(95 \% \mathrm{Cl}) \\
\text { Heterogeneity } \text { Tau }^{2}=62.98 ; \mathrm{Ch}^{2}=91.0 \\
\text { Test for overall effect: } Z=0.33(P=0.74)\end{array}$}} & & 2210 & & & & $100.0 \%$ & $0.91[-4.52,6.34]$ & \\
\hline & & & & & & & & & 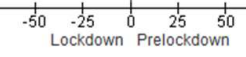 \\
\hline
\end{tabular}

c)

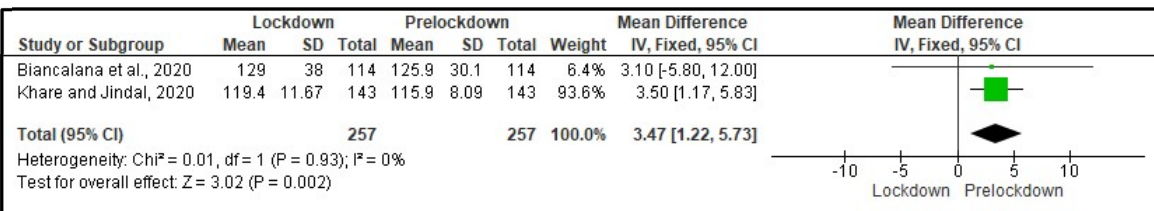

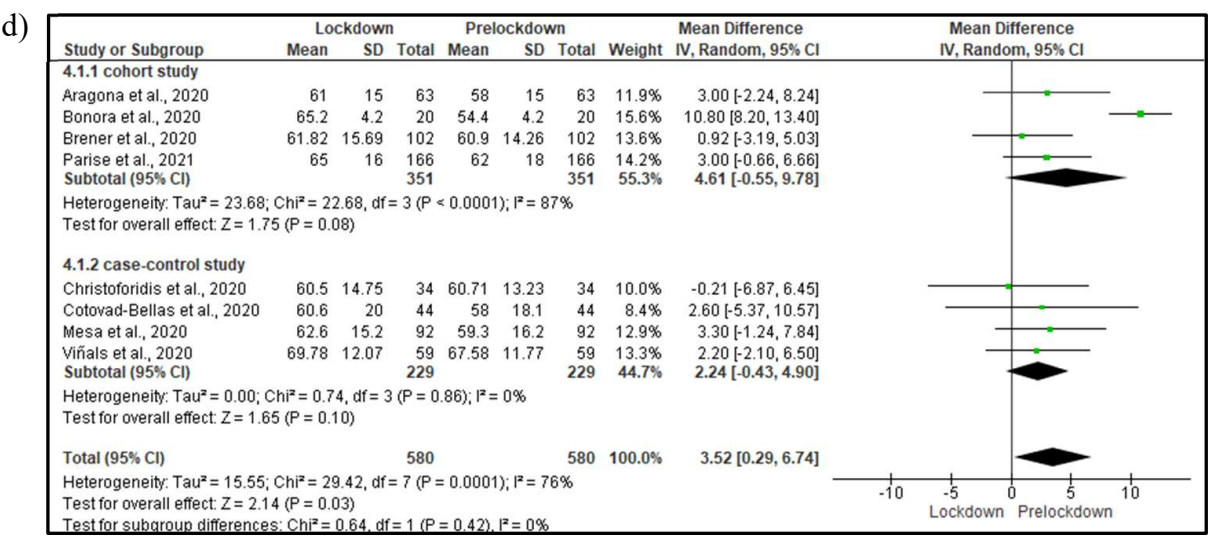

e)

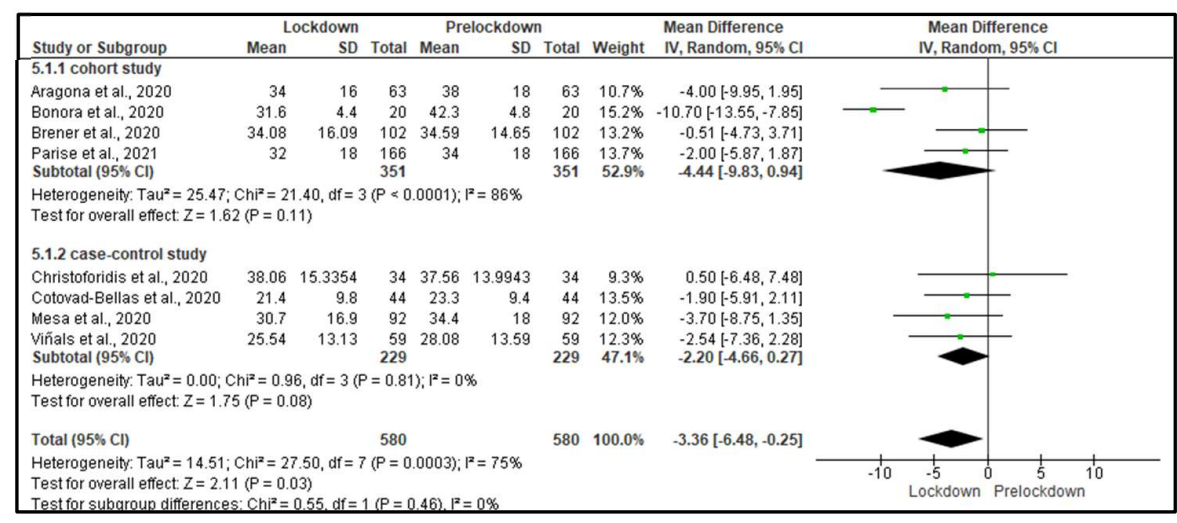

f)

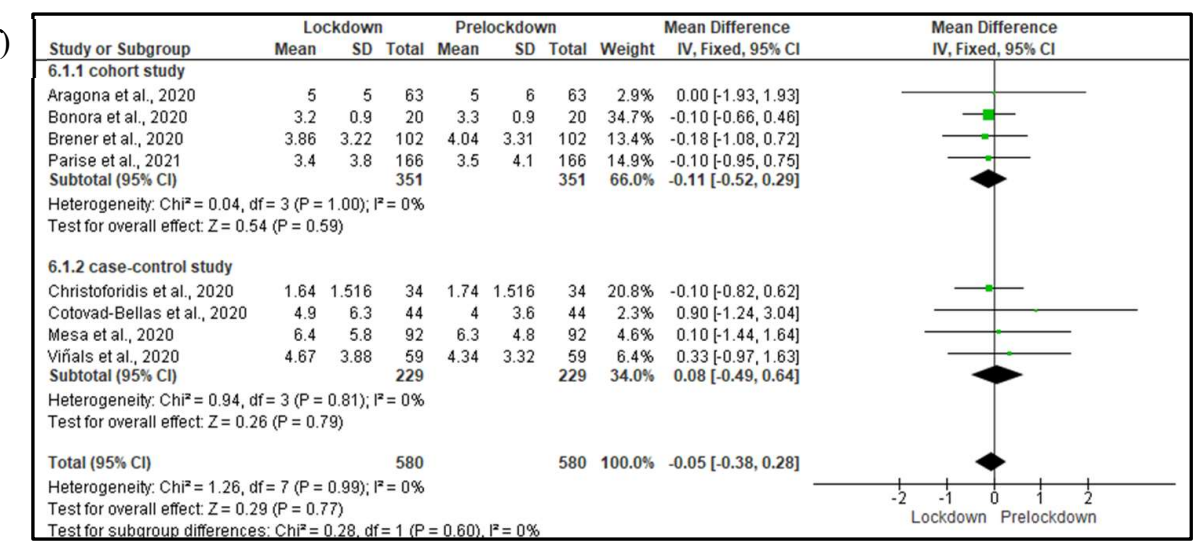

Figure 2 Meta-analyses of a) HbA1c, b) RBG, c) FBG, d) TIR, e) TAR, and f) TBR 


\section{Time Below Range (TBR)}

Data of mean level of TBR was reported in 8 studies [22,24,26,28,29,33-35] among T1D patients with a total of 580 participants on each arm (Figure 2). TBR was not significantly affected during the lockdown period ( $\mathrm{MD}=-0.05 \%$ [95\% CI -0.38 to 0.28$], \mathrm{I}^{2}=0 \%$, fixed-effect). Sub-group analysis was performed and insignificant changes in TBR were presented on both cohort studies ( $\mathrm{MD}=-0.11 \%$ [ $95 \% \mathrm{CI}-0.52$ to 0.29], $\mathrm{I}^{2}=0 \%$, fixed-effect) and case-control studies ( $\mathrm{MD}=0.08 \%$ [95\% $\mathrm{CI}-0.49$ to 0.64$]$, fixed-effect).

a)

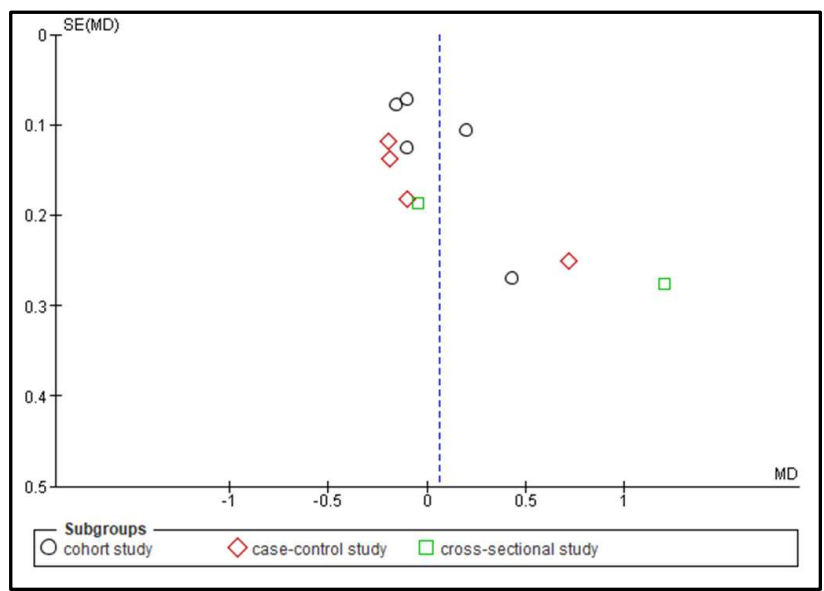

b)

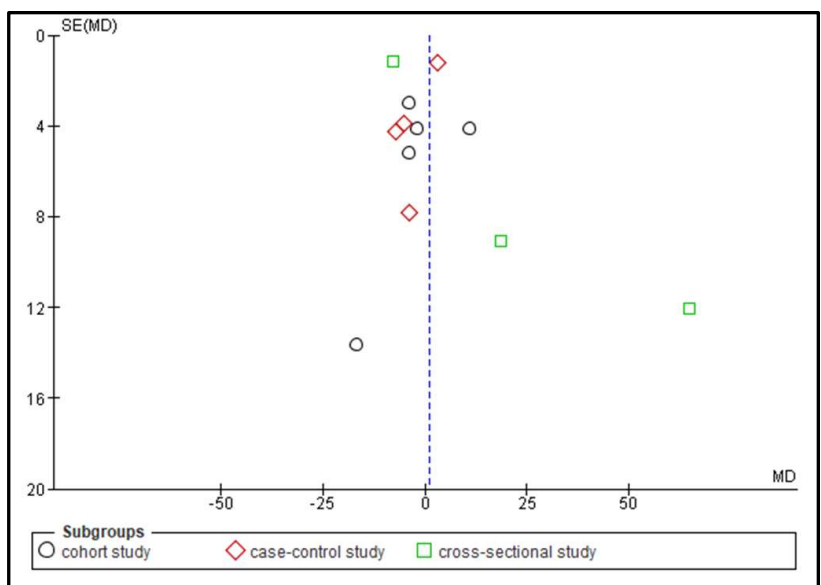

c)

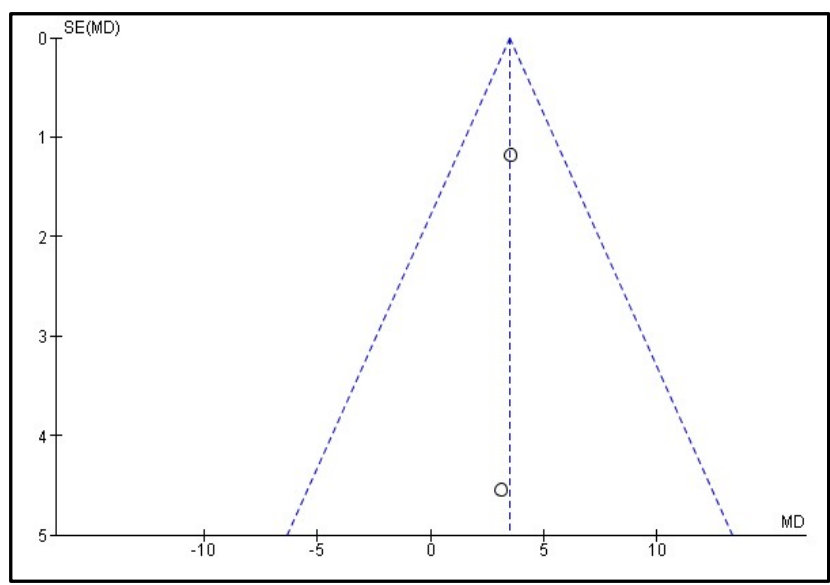

d)

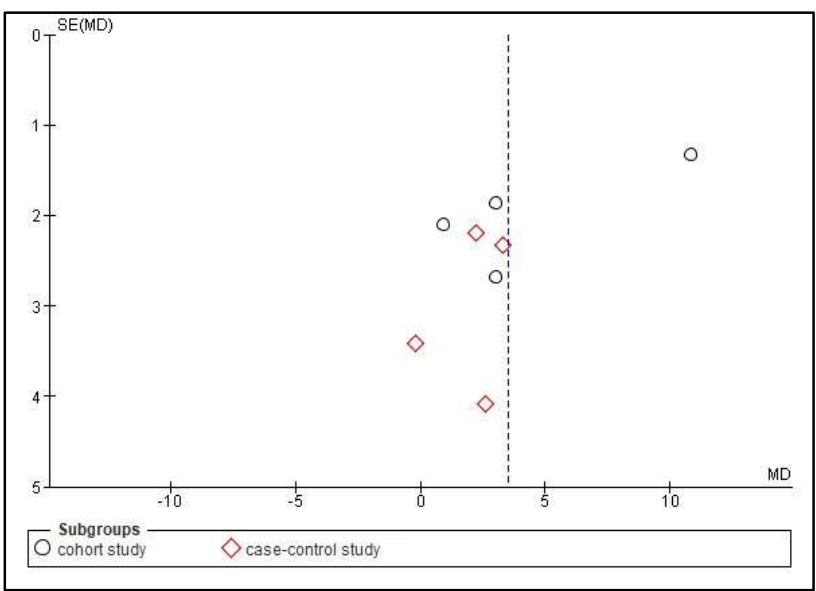

e)

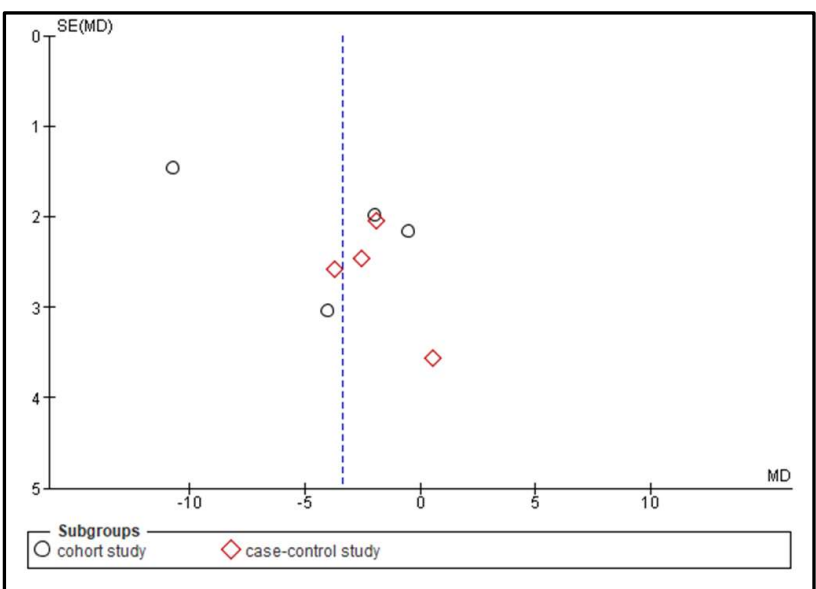

f)

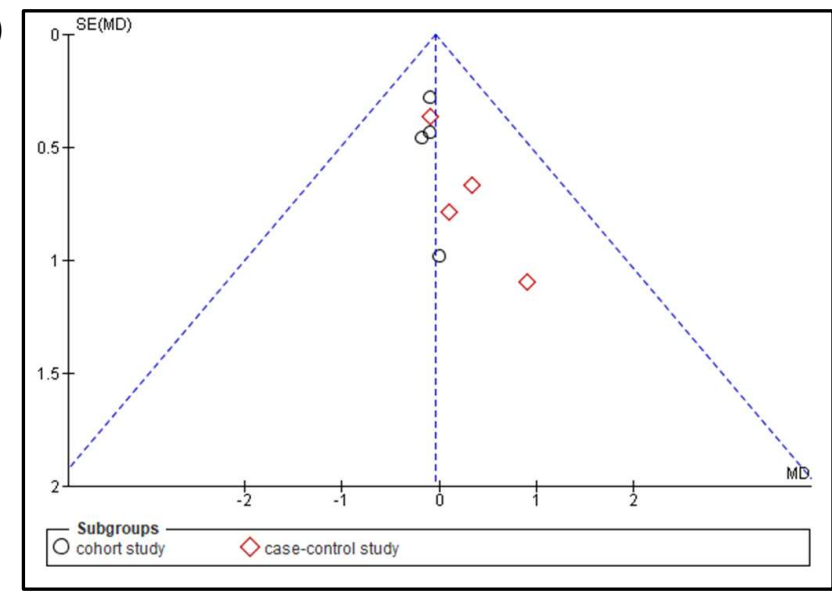

Figure 3 Funnel plot of a) HbA1c, b) RBG, c) FBG, d) TIR, e) TAR, and f) TBR outcomes.

\section{Sensitivity Analysis}


Heterogeneity of variance was found in $\mathrm{HbA1c}, \mathrm{RBG}$, TIR, and TAR analysis, thus sensitivity analysis was done to identify the source of heterogeneity using the leave-one-out method on moderate-quality studies. Bonora et al. [33] was found to be the source of heterogeneity in both TAR and TIR. Neither the direction of effect nor the statistical significance was changed when this study was excluded from each outcome. Sensitivity analysis on each sub-group analysis of HbAlc and RBG was unable to provide significant results.

\section{Secondary Outcomes Lipid Control}

Meta-analysis on lipid profile was unable to be performed due to limited data available. An Italian cohort study [23] with 114 Caucasian T2D patients reported significant improvements in total cholesterol and LDL levels during the lockdown $(p<0.05)$ with a slight increase in triglycerides $(p>0.05)$, despite consistent doses and types of medication administered before and during the lockdown. On the contrary, a Turkish case-control study [27] with 85 T2D patients reported a significant increase in triglyceride level $(p<0.05)$ and numeric increases in LDL and HDL $(p>0.05)$, along with a significant increase in body weight. Multinomial logistic regression in Biancalana et al. study [23] and multivariate analysis in Karatas et al. study [27] also revealed disparate outcomes, where one study reported pre-lockdown triglyceride as a predictor of glycemic control during the lockdown period and the other reported that diabetic duration is the independent predictor of both glycemic control (HbAlc) and lipid profile (TG) [OR 1.2, 95\%CI 1.1 $-1.8, \mathrm{p}=0.032$ ]. Karatas et al. [27] reported that increases in body weight, blood glucose, HbA1c, and plasma lipids simultaneously serve as triggers in the expanded risk of cardiovascular diseases.

\section{DISCUSSION}

The results of this systematic review and meta-analysis indicated a significant association between lockdown and changes in FBG, TIR, and TAR among diabetic patients, although insignificant results were also found on the analysis of TBR, HbAlc, and RBG.

The lockdown and quarantine policy, which has been implemented in many countries, deeply impacted everyone's daily life. Changes in diet, sleep, and physical activity, along with psychological stress, identified as the leading cause of short-term worsening in glycemic control. Increased food craving and emotional eating of unhealthy sugary snacks or energy foods, stress-eating, snacking after dinner, reduced dietary limitations, along with sleep disturbance, and reduced physical activity was thought to expand the risk of weight gain during lockdown [38]. However, the hypothesis regarding dysfunctional eating habits related to boredom during the lockdown, which is associated with increased hyperglycemia, was only found in the first few days of the lockdown [6,7,39].

In this review, patients' ability to use glucose monitoring devices properly showed a decent socioeconomic and educational background, which reflected their care for their condition; thus, sufficient glycemic control was expected, both before and during the lockdown. The use of CGM or FGM devices and the application of sensor-augmented insulin pumps (SAP) in diabetic management also independently permit substantial impacts on glycemic control [40].

Lockdown had reckoned to have positive impacts, primarily in patients who previously had good glycemic control. Home confinement indirectly improves glycemic control with sufficient time to focus on the management of diabetes mellitus. Changes in work-related routine had allowed patients to constantly check glucose status, quickly respond to alarms and notifications of the device, and regularly calibrate the monitoring device. With the abundance of leisure, supported by financial considerations and limited access to outside dining, home-based food consumption, healthier diet selection, and alcohol access limitation were profoundly promoted, thus leading to a more precise carbohydrate calculation, organized mealtime, and in-time administration of insulin. Hypothetically, these changes minimize the hypoglycemia and hyperglycemia occurrence pronounced by improved glycemic variability, particularly in patients who stopped working during the lockdown [26,28,29,33,36,39-41].

Previous studies further reported that the TIR value improved by continuous and structured medical assistance through telemedicine and regular glycemic data reports by patients and caregivers. The aforementioned allowed doctors to swiftly respond to any changes in glycemic variability by adjusting 
daily insulin doses according to the uploaded data, particularly in patients who use HCL or the insulin pump system. Thus, the hypoglycemic and hyperglycemic state and the overall glycemic variability could be improved $[24,39,41]$.

Stability in glycemic variability also reflects a good adaptation of patients and caregivers against extreme changes in daily routines and their willingness to maintain glycemic control despite the disruption in almost all aspects of life due to pandemic and lockdown [34]. Improvements on disruptive habits, including the inadequacy of physical exercise, could also be possible regarding the spare time [24,26]. Further increases were reported in patients who retained their routine physical activity at home before and during the lockdown [41]. Physical activity was believed to reduce stress and anxieties regarding pandemics and improve the quality and duration of sleep. A good sleep could result in a more contained behavior leading to stability and improvement in glycemic control [22]. Association between perceived stress scale (PSS) scores and incidence of severe hypoglycemia (TBR $<54 \mathrm{mg} / \mathrm{dL}$ ) also strengthen the urgency of stress reduction [32].

In concordance with glycemic variability analysis, studies on the adult T1D population presented favorable blood glucose and HbA1c values during the lockdown period [22,24-26,28,29,31]. The findings also suggested a reduced dawn phenomenon near the end of the lockdown, which indicates a better glycemic control towards the end of the confinement [22].

However, changes in $\mathrm{HbA1} \mathrm{c}$ and estimated A1c_or glucose management indicator (GMI) which approximated based on average blood glucose measured using CGM values-among T1D and T2D patients were found insignificant [42]. One study found that pediatric patients of T1D presented a higher HbAlc value during the lockdown. The increase is suspected to be correlated with increased BMI, decreased physical activity, and increased screen time [21]. Al-Agha et al. [30] found a decrease in HbA1c. Nonetheless, it was not followed by improvements in RBG and other related factors, including BMI, diet, and sedentary habits. Difficulty in acquiring insulin, stress, and mood deterioration was also reported [30]. Thus, both studies assumed a regression of a previously healthy lifestyle at diabetic patients had occurred during the lockdown period.

In addition, HbA1c analysis of T2D patients showed a similar increase as the pediatric T1D population $[23,25,27]$, although the increase was statistically insignificant since it requires a longer time to evaluate any changes in the HbA1c; some of the studies were carried out in less than 12 weeks [25,27]. Factors directly correlated to the decline were increased triglyceride, carbohydrate-rich diet, body weight, perceived stress, and decreased physical activity [23,25,27]. Other indirect factors introduced include limited access to insulin and glucose strips, lower socioeconomic status, and limitation of mobility and income $[30,31,34]$.

Increased number of home glucose-monitoring seemed to be the plausible objective of the HbA1c improvement. More frequent glucose monitoring would lead to a consistent in-time insulin administration required for an excellent glycemic index [44]. Therefore, regardless of the deterioration in other factors, regular insulin therapy and constant glucose monitoring seemed to impact HbAlc significantly.

Several studies indeed implied contradictory results, possibly due to the different demographics of the patients. Financial and work instability in low socioeconomic populations was reported to lead to the inability of patients to provide a good diet and escalation of perceived stress due to fears and anxieties regarding the adequacy of food, which exacerbated the worsening of glycemic control. The economic crisis during the pandemic due to increasing rates of unemployment and financial insecurity had also compromised people in lower socioeconomic positions, particularly in younger patients. Home confinement, work from home regulation, and closure of public places such as parks and sports centers gave rise to a drastic decline in physical activity. As a result, it may limit patient access to medical treatment, decline the number of clinical visits, and increase the circumstance of substandard care, hence further impacting glycemic control [6,7,32,34,40,44]. Christoforidis et al. [35] also reported alterations in meal timing to a looser routine with frequent late-night eating and a significantly increased percentage of the late first meal of the day around $10.00 \mathrm{a} . \mathrm{m}$. Furthermore, increased basal insulin dose was also reported due to an increasingly sedentary lifestyle, although the total daily insulin dose was not increased, as the bolus insulin dose was reduced [35]. 
Lastly, we found that most lipid control parameters among T2D patients were generally under control and not affected by lockdown. Interestingly, the elevation of mean triglyceride level was reported in both the Turkish case-control study and Italian cohort study, while only the latter showed significance $[23,27]$. Since none of the participants reduced or changed the course of treatment during the lockdown, the alteration of lipid profile in diabetic patients was merely associated with changes in eating habits, regular physical activity, and controlled dietary approaches [45]. A survey in Spain of T2D patients showed that during the pandemic, the behavior of craving snacks and sugary foods and decreased physical activity tended to increase and exacerbated by the lockdown policies [11]. Additionally, poor socioeconomic conditions amidst pandemic changed dietary patterns and created tendencies that promoted obesity, including the surge of more accessible and affordable fast foods. Limited mobility and decreased physical activity can increase daily sitting time from 5 to 8 hours per day, leading to reduced calorie expenditure [46].

A previous observational study explained that a high-carbohydrate diet tends to increase fasting triglyceride levels and is associated with higher mortality rates [47]. Triglycerides are closely related to non-esterified fatty acids and could have hazardous effects on glucose homeostasis due to the lipotoxicity mechanism; even serum triglycerides independently could represent a risk factor for cardiovascular and kidney diseases $[48,49]$.

\section{Limitation}

We included retrospective and prospective studies in this review due to limited studies available since any controlled studies were barely possible to conduct amid the lockdown period. Diversities in duration and technical approach of lockdown implementation between countries worldwide had resulted in the heterogeneity of methods and results in this review, which along with the brief study period, had given rise to limitations in data availability. Thus, particular outcomes could not be thoroughly analyzed. Further study with an extended period, larger population, and observation of the after-lockdown findings could advance the significance of reviews on similar topics. Analysis of lipid profile should be further deepened in a more focused setting. To the best of our knowledge, until the manuscript publication submission, this is the first systematic review observing the comparison of glycemic control parameters and lipid profile of diabetic patients before and during COVID-19 lockdown. The inclusion of both T1D and T2D patients with various types of study designs including cohort, case-control, and cross-sectional studies from various countries worldwide supported the superiority of this review.

\section{CONCLUSION}

Lockdown enforcement was not proven to worsen the glycemic control parameter in diabetic patients. However, the results of this study could be considered as references in advancing the care and management of diabetic patients, particularly during confinements (i.e., quarantine, lockdown) since poor control of the parameters may promote the progression of diabetic complications and increase vulnerability during the COVID-19 pandemic. Besides, the finding of this study reveal a good potential of telemedicine for patient control and provide a scientific basis for its development. Furthermore, this study provided evidence that sudden short-term changes in lifestyle might affect glycemic control. Therefore, a healthy lifestyle and proper diabetes management should remain enforced in patients with diabetes besides pharmacological care.

\section{Author Contributions}

All authors conceived the main conceptual idea. IAW and NRP independently conducted literature search and selection and title and abstract screening on each article. IAW, NRP, and NFS extracted the study data. IAW and NFS independently performed quality assessment. ESA and TK carried out the statistical analysis. MAW and MRW drafted the manuscript. LD and HN encouraged authors to investigate the impact of COVID-19 lockdown and supervised the findings of this work. All authors discussed the results and contributed to the final manuscript. 


\section{REFERENCES}

1. Alshareef R, Al Zahrani A, Alzahrani A, Ghandoura L. Impact of the COVID-19 lockdown on diabetes patients in Jeddah, Saudi Arabia. Diabetes Metab Syndr 2020;14:1583-1587.

2. Cambridge University. Lockdown. Cambridge Press University. Available from: https://dictionary.cambridge.org/dictionary/english/lockdown (Updated of April 24, 2021).

3. Ontario. COVID-19 response framework: keeping Ontario safe and open. Available from: https://www.ontario.ca/page/COVID-19-response-framework-keeping-ontario-safe-and-open (Updated of April 24, 2021)

4. Yadav AK. Impact of lockdown to control over Novel Coronavirus and COVID-19 in India. J Family Med Prim Care 2020;9:5142-5147.

5. Alfano V, Ercolano S. The Efficacy of Lockdown Against COVID-19: A Cross-Country Panel Analysis. Appl Health Econ Health Policy 2020;18:509-517.

6. Ismail LC, Osaili TM, Mohamad MN, Al Marzouqi A, Jarrar AH, Abu Jamous DO et al. Eating Habits and Lifestyle during COVID-19 Lockdown in the United Arab Emirates: A Cross-Sectional Study. Nutrients 2020;12:3314.

7. Di Renzo L, Gualtieri P, Pivari F, Soldati L, Attinà A, Cinelli G et al. Eating habits and lifestyle changes during COVID-19 lockdown: An Italian survey. J Transl Med 2020;18:229.

8. Saqib MAN, Siddiqui S, Qasim M, Jamil MA, Rafique I, Awan UA et al. Effect of COVID-19 lockdown on patients with chronic diseases. Diabetes Metab Syndr 2020;14:1621-1623.

9. Consonni M, Telesca A, Grazzi L, Cazzato D, Lauria G. Life with chronic pain during COVID-19 lockdown: the case of patients with small fibre neuropathy and chronic migraine. Neurol Sci. 2021;42:389-397.

10. Ghosh A, Arora B, Gupta R, Anoop S, Misra A. Effects of nationwide lockdown during COVID19 epidemic on lifestyle and other medical issues of patients with type 2 diabetes in north India. Diabetes Metab Syndr. 2020;14:917-920.

11. Ruiz-Roso MB, Knott-Torcal C, Matilla-Escalante DC, Garcimartín A, Sampedro-Nuñez MA, Dávalos A et al. COVID-19 Lockdown and Changes of the Dietary Pattern and Physical Activity Habits in a Cohort of Patients with Type 2 Diabetes Mellitus. Nutrients. 2020;12:2327.

12. Monnier L, Colette C, Owens DR. Glycemic variability: the third component of the dysglycemia in diabetes. Is it important? How to measure it?. J Diabetes Sci Technol. 2008;2:1094-1100.

13. Warraich HJ, Rana JS. Dyslipidemia in diabetes mellitus and cardiovascular disease. Cardiovasc Endocrinol. 2017;15:27-32.

14. Wolff D, Nee S, Hickey NS, Marschollek M. Risk factors for Covid-19 severity and fatality: a structured literature review. Infection. 2021;49:15-28.

15. Guan WJ, Liang WH, Zhao Y, Liang HR, Chen ZS, Li YM et al. Comorbidity and its impact on 1590 patients with COVID-19 in China: a nationwide analysis. Eur Respir J. 2020;55:2000547.

16. Banerjee M, Chakraborty S, Pal R. Diabetes self-management amid COVID-19 pandemic. Diabetes Metab Syndr. 2020;14:351-354.

17. Page MJ, McKenzie JE, Bossuyt PM, Boutron I, Hoffmann TC, Mulrow CD et al. The PRISMA 2020 statement: an updated guideline for reporting systematic reviews. BMJ. 2021;372:n71.

18. Wafa IA, Pratama NR, Sofia NF, Anastasia ES, Konstantin T, Wijaya MA, Wiyono MR, Djuari L. Impact of COVID-19 lockdown on the metabolic control parameters in patients with diabetes mellitus: systematic review and meta-analysis. PROSPERO 2021 CRD42021252321. Available from: https://www.crd.york.ac.uk/prospero/display record.php?ID=CRD42021252321

19. Armijo-Olivo S, Stiles CR, Hagen NA, Biondo PD, Cummings GG. Assessment of study quality for systematic reviews: a comparison of the Cochrane Collaboration Risk of Bias Tool and the Effective Public Health Practice Project Quality Assessment Tool: methodological research. J Eval Clin Pract 2012;18:12-8.

20. Battelino T, Danne T, Bergenstal RM, Amiel SA, Beck R, Biester T et al. Clinical Targets for Continuous Glucose Monitoring Data Interpretation: Recommendations From the International Consensus on Time in Range. Diabetes Care 2019;42:1593-1603. 
21. Alaqeel A, Aljuraibah F, Alsuhaibani M, Huneif M, Alsaheel A, Al Dubayee M et al. The Impact of COVID-19 Pandemic Lockdown on the Incidence of New-Onset Type 1 Diabetes and Ketoacidosis Among Saudi Children. Front Endocrinol (Lausanne) 2021;12:669302.

22. Aragona M, Rodia C, Bertolotto A, Campi F, Copelli A, Giannarellu R et al. Type 1 diabetes and COVID-19: The "lockdown effect". Diabetes Res Clin Pract 2020;170:108468.

23. Biancalana E, Parolini F, Mengozzi A, Solini A. Short-term impact of COVID-19 lockdown on metabolic control of patients with well-controlled type 2 diabetes: a single-centre observational study. Acta Diabetol. 2021;58:431-436.

24. Parise M, Tartaglione L, Cutruzzolà A, Maiorino MI, Esposito K, Pitocco D et al. Teleassistance for Patients With Type 1 Diabetes During the COVID-19 Pandemic: Results of a Pilot Study. J Med Internet Res 2021;23:e24552

25. Ruissen MM, Regeer H, Landstra CP, Schroijen M, Jazet I, Nijhoff MF et al. Increased stress, weight gain and less exercise in relation to glycemic control in people with type 1 and type 2 diabetes during the COVID-19 pandemic. BMJ Open Diabetes Res Care 2021;9:e002035.

26. Cotovad-Bellas L, Tejera-Pérez C, Prieto-Tenreiro A, Sánchez-Bao A, Bellido-Guerrero D. The challenge of diabetes home control in COVID-19 times: Proof is in the pudding. Diabetes Res Clin Pract 2020;168:108379.

27. Karatas S, Yesim T, Beysel S. Impact of lockdown COVID-19 on metabolic control in type 2 diabetes mellitus and healthy people. Prim Care Diabetes 2021 Jun;15:424-427.

28. Mesa A, Viñals C, Pueyo I, Roca D, Vidal M, Giménez M et al. The impact of strict COVID-19 lockdown in Spain on glycemic profiles in patients with type 1 Diabetes prone to hypoglycemia using standalone continuous glucose monitoring. Diabetes Res Clin Pract 2020;167:108354.

29. Viñals C, Mesa A, Roca D, Vidal M, Pueyo I, Conget I et al. Management of glucose profile throughout strict COVID-19 lockdown by patients with type 1 diabetes prone to hypoglycaemia using sensor-augmented pump. Acta Diabetol 2021;58:383-388.

30. Al Agha AE, Alharbi RS, Almohammadi OA, Yousef SY, Sulimani AE, Alaama RA. Impact of COVID-19 lockdown on glycemic control in children and adolescents. Saudi Med J 2021;42:44-48.

31. Verma A, Rajput R, Verma S, Balania VKB, Jangra B. Impact of lockdown in COVID 19 on glycemic control in patients with type 1 Diabetes Mellitus. Diabetes Metab Syndr 2020;14:12131216.

32. Barchetta I, Cimini FA, Bertoccini L, Ceccarelli V, Spaccarotella M, Baroni MG et al. Effects of work status changes and perceived stress onglycaemiccontrol in individuals with type 1 diabetes during COVID-19 lockdown in Italy. Diabetes Res Clin Pract 2020;170:108513.

33. Bonora BM, Boscari F, Avogaro A, Bruttomesso D, Fadini GP. Glycaemic Control Among People with Type 1 Diabetes During Lockdown for the SARS-CoV-2 Outbreak in Italy. Diabetes Ther 2020;11:1369-1379.

34. Brener A, Mazor-Aronovitch K, Rachmiel M, Level N, Barash G, Pinhas-Hamiel O et al. Lessons learned from the continuous glucose monitoring metrics in pediatric patients with type 1 diabetes under COVID-19 lockdown. Acta Diabetol 2020;57:1511-1517.

35. Christoforidis A, Kavoura E, Nemtsa A, Pappa K, Dimitriadou M. Coronavirus lockdown effect on type 1 diabetes management on children wearing insulin pump equipped with continuous glucose monitoring system. Diabetes Res Clin Pract 2020;166:108307.

36. Potier L, Hansel B, Larger E, Gautier JF, Carreira D, Assemien R et al. Stay-at-Home Orders During the COVID-19 Pandemic, an Opportunity to Improve Glucose Control Through Behavioral Changes in Type 1 Diabetes. Diabetes Care 2021;44:839-843.

37. Khare J, Jindal S. Observational study on Effect of Lock Down due to COVID 19 on glycemic control in patients with Diabetes: Experience from Central India. Diabetes Metab Syndr 2020;14:1571-1574.

38. Zachary Z, Brianna F, Brianna L, Garrett P, Jade W, Alyssa D et al. Self-quarantine and weight gain related risk factors during the COVID-19 pandemic. Obes Res Clin Pract 2020;14:210-216. 
39. Longo M, Caruso P, Petrizzo M, et al. Glycemic control in people with type 1 diabetes using a hybrid closed loop system and followed by telemedicine during the COVID-19 pandemic in Italy. Diabetes Res Clin Pract 2020;169:108440.

40. Di Dalmazi G, Maltoni G, Bongiorno C, Tucci L, Di Natale V, Moscatiello S et al. Comparison of the effects of lockdown due to COVID-19 on glucose patterns among children, adolescents, and adults with type 1 diabetes: CGM study. BMJ Open Diabetes Res Care 2020;8:e001664.

41. Tornese G, Ceconi V, Monasta L, Carletti C, Faleschini E, Barbi E. Glycemic Control in Type 1 Diabetes Mellitus During COVID-19 Quarantine and the Role of In-Home Physical Activity. Diabetes Technol Ther 2020;22:462-467.

42. Bergenstal RM, Beck RW, Close KL, Grunberger G, Sacks DB, Kowalski A et al. Glucose Management Indicator (GMI): A New Term for Estimating A1C From Continuous Glucose Monitoring. Diabetes Care 2018;41:2275-2280.

43. DiMeglio LA, Evans-Molina C, Oram RA. Type 1 diabetes. Lancet 2018;391:2449-2462.

44. Dover AR, Ritchie A, McKnight A, Strachan WJ, Zammitt NN, Wake DJ et al. Assessment of the effect of the COVID-19 lockdown on glycaemic control in people with type 1 diabetes using flash glucose monitoring. Diabet Med 2020;38:e14374.

45. Doshmangir P, Jahangiry L, Farhangi MA, Doshmangir L, Faraji L. The effectiveness of theoryand model-based lifestyle interventions on HbA1c among patients with type 2 diabetes: a systematic review and meta-analysis. Public Health 2018;155:133-141.

46. Ammar A, Brach M, Trabelsi K, Chtourou H, Boukhris O, Masmoudi L et al. Effects of COVID19 Home Confinement on Eating Behaviour and Physical Activity: Results of the ECLB-COVID19 International Online Survey. Nutrients 2020;28;12:1583.

47. Jung CH, Choi KM. Impact of High-Carbohydrate Diet on Metabolic Parameters in Patients with Type 2 Diabetes. Nutrients 2017;9:322.

48. Seghieri M, Tricò D, Natali A. The impact of triglycerides on glucose tolerance: Lipotoxicity revisited. Diabetes \& metabolism 2017;43:314-322.

49. Marston NA, Giugliano RP, Im K, Silverman MG, O'Donoghue ML, Wiviott SD et al. Association Between Triglyceride Lowering and Reduction of Cardiovascular Risk Across Multiple LipidLowering Therapeutic Classes: A Systematic Review and Meta-Regression Analysis of Randomized Controlled Trials. Circulation 2019;140:1308-1317 